\title{
Morning melatonin ingestion and diurnal variation of short-term maximal performances in soccer players
}

\author{
K Ghattassi ${ }^{1}$, O Hammouda ${ }^{2}$, A Graja ${ }^{1}$, N Boudhina ${ }^{1}$, H Chtourou ${ }^{1}$, \\ S Hadhri ${ }^{3}$, T Driss ${ }^{2}$, N Souissi ${ }^{1,4}$ \\ ${ }^{1}$ Research Laboratory “Sport Performance Optimization”, National Center of Medicine \\ and Sciences in Sport (CNMSS), Tunis, Tunisia \\ ${ }^{2}$ Research Center on Sport and Movement (CeRSM, EA 2931), Sciences and Techniques \\ of the Physical and Sporting Activities (UFR STAPS), University of Paris Ouest Nanterre La Defense, \\ Nanterre, France \\ ${ }^{3}$ High Institute of Sport and Physical Education, University of Sfax, Sfax, Tunisia \\ ${ }^{4}$ High Institute of Sport and Physical Education, Ksar-Saïd, Manouba University, Tunis, Tunisia
}

Received: July 2, 2015

Accepted: January 27, 2016

\begin{abstract}
Aim: Very few studies have investigated the temporal specificity of melatonin (MEL) ingestion upon short-term maximal athletic performances. The aim of the present study was to explore the effect of morning MEL ingestion on cognitive and physical performances measured in the afternoon. Methods: Twelve soccer players from a Tunisian squad $(17.9 \pm 1.3$ years, $1.74 \pm 0.06 \mathrm{~m}$ and $62.0 \pm 8.8 \mathrm{~kg})$ participated in the present study. They performed two testing sessions at 08:00 h, 12:00 h and 16:00 h after either MEL (5mg) or placebo (PLA) ingestion, in a randomized order. During each period, the participants performed the following cognitive and physical tests: reaction time and vigilance tests, medicine-ball throw (MBT), five jumps, handgrip strength (HG), and agility tests. Results: cognitive and physical performances were significantly higher at 16:00 h compared to 08:00 h during the two conditions $(\mathrm{p}<0.05)$. Moreover, performances of MBT and HG were lower in the morning with MEL in comparison to PLA $(\mathrm{p}<0.05)$. However, MEL ingestion did not affect physical and cognitive performances measured at 12:00 h and 16:00 h. Conclusion: morning MEL ingestion has no unfavourable effect on afternoon physical and cognitive performances in soccer players.
\end{abstract}

Keywords: melatonin, diurnal variation, athletic performance, soccer

Melatonin (N-acetyl-5-methoxytryptamine) (MEL) is an indole mainly produced by the pineal gland in all vertebrates following a light dark cycle. MEL, often considered as a chronobiotic (12), was initially investigated in terms of its role in endocrine physiology regulating circadian and sometimes seasonal rhythms (22). Moreover, it has been shown that exogenous MEL can improve sleep quality and alleviate jet lag symptoms after transmeridian travels (5). In addition to the control of the biological rhythms, it is known that exogenous MEL administration has clear hypothermic effects (10).

Otherwise, some reciprocal benefices have been evidenced between exercise and MEL production in humans (10). Therein, it has been previously shown that diurnal MEL ingestion

Corresponding author: Omar Hammouda, $\mathrm{PhD}$

Research Center on Sport and Movement (CeRSM, EA 2931), Sciences and Techniques of the Physical and Sporting Activities (UFR STAPS), University of Paris Ouest Nanterre La Defense, 200 avenue de la République, 92001, Nanterre, France

Phone: + 33 782931995; E-mail: hammouda.o@u-paris10.fr

0231-424X/\$ 20.00 C 2016 Akadémiai Kiadó, Budapest 
$(0.1-10 \mathrm{mg})$ in young healthy men could achieve sleepiness, fatigue, alertness, weaker balance and poorer coordination in locomotion (9). Furthermore, Dollins et al. (8) reported that midday MEL ingestion (i.e., $10-80 \mathrm{mg}$ ) affected reaction time and decreased subjective alertness and the number of correct responses on a vigilance task. In the same way, Jones (15) concluded that MEL administration $(5 \mathrm{mg}$ ) at $11: 45 \mathrm{~h}$ with repeated performance tests at 13:00 $\mathrm{h}$ and 15:00 $\mathrm{h}$ adversely affected choice reaction time, subjective alertness, short-term memory, and mean heart rate measured during a 4-km cycling time trial. However, the 4-km cycling time was not affected. Similarly, Atkinson et al. (3) concluded that, MEL (5 mg) ingested at the same time as Jones (15) with repeated measures at 13:00 h and 17:00 h, affected cognitive performances but not the $4 \mathrm{~km}$ cycling time.

The discrepancy between previous studies could be explained by various factors (i.e., time of ingestion, dose of MEL, fitness level, exercise intensity and modality, difference in lighting conditions) (4). In this sense, a time-of-day effect of MEL administration (5mg) at $12: 00,17: 00,19: 00$, and $21: 00 \mathrm{~h}$ has been reported using the ultra short (7"/13") sleep schedule paradigm (21).

Otherwise, it has been recently found that soccer players perform at an optimum between 16:00 $\mathrm{h}$ and 20:00 $\mathrm{h}$ when not only soccer-specific skills (e.g., juggling performance, wallvolley test, etc.) but also measures of physical (e.g., vertical jump, grip strength, etc.) and cognitive (i.e., alertness and reaction time) performances are at their peak (18). Moreover, professional soccer matches are played at various times-of-day, ranging from morning kickoffs to night-time competitions under floodlights. Nevertheless, a large inter-individual variation in circadian rhythmicity has been observed (23). For example, reductions in core temperature are variable, ranging from 0.01 to $0.38^{\circ} \mathrm{C}$. In this way, hypothermic effects have been found to be still present 4-7 h after MEL ingestion (14).

The aim of this study was to investigate the effect of morning MEL ingestion on subsequent diurnal variations of mental and short-term maximal performances in soccer players. We hypothesized that morning MEL ingestion could affect morning physical and cognitive performances but not afternoon ones in soccer players. For this aim, some soccer specific tests were assessed (reaction time and vigilance tests, medicine-ball throw (MBT), five jumps (5JT), hand grip strength (HG), and modified agility tests (MAT). Moreover, core temperature was measured to investigate if hypothermic effects induced by morning MEL ingestion could affect subsequent performances.

\section{Methods}

\section{Participants}

Twelve soccer players $(17.9 \pm 1.3$ years, $1.74 \pm 0.06 \mathrm{~m}$ and $62.0 \pm 8.8 \mathrm{~kg}$; mean $\pm \mathrm{SD})$ from a Tunisian League 3 squad participated in the present study. After receiving a description of the protocol, its benefits and possible risks, each volunteer (and parents/tutors for the minors) provided a written informed consent. The experimental design was approved by the Ethical Committee of the University Hospital (CHU) of Farhat Hached Sousse Tunisia and met the ethical standards of the Declaration of Helsinki. They were non-smokers, did not consume caffeine or alcoholic beverages that could enhance or compromise wakefulness. None suffered from lower-limb injuries or muscle soreness during the experiment. To ensure that participants were all of a "moderately morning type" $(n=7)$ or "neither type" $(n=5)$, they were selected based on their responses to the Horne and Östberg's self-assessment 
questionnaire (13). Furthermore, during the week prior to the experiment, participants were familiarized with the experimental procedures in order to minimize the learning effect during the course of the study. They performed two test sessions, in a randomized order, over 2 days with three test periods per day, allowing a recovery period of $36 \mathrm{~h}$ in between. One MEL ingestion session (i.e., $5 \mathrm{mg}$ (Jamieson Laboratories Toronto, Canada) according to the dosing advices of Arendt and Deacon (2) and one PLA session. Throughout the experimental period, participants were requested to maintain their habitual physical activity and to avoid strenuous activity during the $24 \mathrm{~h}$ before the test sessions.

During each testing session, participants were requested to attend the laboratory at 20:00 h. Body mass (Tanita, Tokyo, Japan) and height (Stadiometer, QuickMedical, USA) were recorded. Participants were asked to keep, as closely as possible, their usual sleeping habits, with at least $7 \mathrm{~h}$ of night sleep preceding each test session (sleeping between 23:00 and 07:00 $\pm 1: 00 \mathrm{~h}$ ). Before the morning test period, they were instructed to wake up at 06:00 h. They were fasting and allowed to drink only one glass of water to avoid the effects of postprandial thermogenesis (7). Moreover, they were requested to ingest a standardized breakfast and lunch meals at least $3 \mathrm{~h}$ before the midday and afternoon test period respectively, as recommended by Bougard et al. (7). After meals, only water was allowed ad libitum. The overall daily energy intake goal was set at $10.5 \mathrm{MJ}$ per capita/day. MEL or PLA were ingested in capsule in the morning at 07:30 h, 30 min before starting the test battery, in a double-blind randomized design. Moreover, the 30-min duration between MEL ingestion and exercise was chosen accordingly to the recent finding showing that similar doses of MEL (6 mg), ingested 30 min prior to intense exercise reverses oxidative stress, improves immunological defenses and lipid metabolism in soccer players (16).

During each session, oral temperature was measured with a calibrated and valid digital clinical thermometer (Omron, Paris, France; accuracy: $0.05^{\circ} \mathrm{C}$ ) inserted sublingually for at least 3 min with the participant in a seated resting position just before physical tests. Then, cognitive and physical tests were done according to the following order: reaction time, vigilance, MBT, 5JT, HG, and MAT were repeated at three different times of day $(08: 00 \mathrm{~h}$, 12:00 $\mathrm{h}$ and 16:00 h). Tests were chosen based on previous works on cognitive and short-term physical performances specific to soccer players $(11,16)$. All the participants stayed in the laboratory during all the experimental period (i.e., reading books, doing homework). The study design was realized in accordance with the design of Dollins et al. (8), who successfully studied the effects of MEL on cognitive performances. Moreover, repeated performance measures were designed according to the previous studies about the effect of diurnal MEL ingestion on cognitive and physical performances $(3,15)$. All the participants were already familiar with the testing procedures as it takes part of their usual fitness assessment program. The light intensity of the field was near 120,1100 and 900 lux, respectively at 08:00 h, 12:00 $\mathrm{h}$ and 16:00 $\mathrm{h}$ during the two test sessions. During the entire experimental period, the mean ambient temperature and relative humidity of the field were kept stable $\left(18.9 \pm 1.2^{\circ} \mathrm{C}\right.$ and $37.5 \pm 7.3 \%$, respectively).

\section{Measurements and protocols}

Cognitive performances:

Simple reaction time was recorded from 10 trials in response to a visual stimulus presented on a computer screen. The response requires participants to press a button with the index finger when a visual stimulus (red point) appears on the screen. Reaction time was recorded 
in (ms) using an electronic timing device (Heuer, Germany). The vigilance test is a test barrage that means the subject has to circulate a figure composed of three numbers in a period of $1 \mathrm{~min}$. In this study, the total circling number in a period of 1 min was taken into account to determine the performance for each participant.

\section{Physical testing}

\section{Medicine-ball throws (MBT)}

A $45^{\circ}$ incline bench was used for the MBT test to facilitate an optimal trajectory of $45^{\circ}$ and to result in arm to torso angles. All the participants used 4-kg medicine balls. A measuring tape was placed on the lawn with the near end positioned under the frame of the bench to anchor it. All participants were allowed two MBT throws. The recorded score for this test was the best distance, which reflects upper body power.

\section{Five jump test (5JT)}

The 5JT consists of 5 consecutive strides with joined feet position at the start and end of the jumps to estimate lower body power. It was performed on the grass with the players equipped with appropriate soccer boots. From the starting joined feet position, they had to directly jump to the front with a leg of their choice. After the first 4 strides, i.e., alternating left and right feet for twice each, they had to perform the last stride and end the test again with joined feet. Performance was measured with a tape measure from the front edge of the player's feet at the starting position to the rear edge of the feet at the final position.

\section{Handgrip strength $(H G)$}

Handgrip strength of the dominant hand was measured with a calibrated dynamometer (T.K.K. 5401; Takei, Tokyo, Japan). Participants were standing comfortably with the shoulder adducted. The dynamometer was held freely without support; it was not touching the participant's trunk. The position of the hand remained constant with a downward direction. The palm did not flex on the wrist joint. Participants performed two trials with $1 \mathrm{~min}$ in between, and the best performance was used for further analysis.

\section{Modified Agility T-test (MAT)}

MAT was used to determine speed with changes of direction such as forward sprinting, left and right shuffling, and backpedaling (20). Participant began with both feet behind the starting line-A. At the own discretion, each participant sprinted forward to cone-B and touched the base of it with the right hand. Facing forward and without crossing feet, the participants shuffled to the left to cone-C and touched its base with the left hand. They then shuffled to the right to cone-D and touched its base with the right hand. They shuffled back to the left to cone-B and touched its base. Finally, they ran backward as quickly as possible and returned to line-A (Fig. 1). The recorded score for this test was the better time of the two last trials. The MAT performances were recorded using an electronic timing system (Globus, Microgate; SARL, Italy).

\section{Statistical analysis}

Data were presented as mean \pm standard error (SE). All statistical tests were processed using STATISTICA10 Software (StatSoft, France). The Shapiro-Wilk test revealed that the data were normally distributed. Dependent variables were analyzed with a two-factor (treatment 
and time-of-day) generalized linear model (GLM) with repeated measures for the two factors. Both factors were 'within-subjects' by definition and included two 'levels' (MEL/PLA) in the treatment factor and three 'levels' (morning, midday, afternoon) in the time-of-day factor). The main effects of treatment and time-of-day were examined together with the interaction between these factors. When appropriate, significant differences among means were tested using the Tukey post-hoc test. A probability level of 0.05 was selected as the criterion for statistical significance.

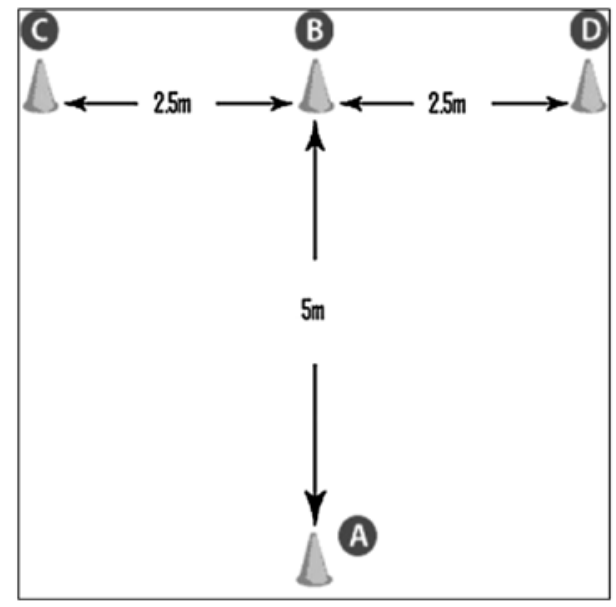

Fig. 1. Agility test (MAT) modified by Sassi et al., (2009)

\section{Results}

\section{Cognitive performances}

Table 1 shows the statistical results for the cognitive performances at the three times of day. There was a significant effect of daytime $(\mathrm{F}=12.95 ; \mathrm{p}<0.05)$ on reaction time. Reaction time performances were significantly higher at 16:00 $\mathrm{h}$ than 12:00 $\mathrm{h}$ and 08:00 $\mathrm{h}$ independently of the treatment $(\mathrm{p}<0.05)$. Moreover, the treatment factor was significant $(\mathrm{F}=4.46 ; \mathrm{p}<$ 0.05). Reaction time performances were significantly lower with MEL in comparison to PLA at 08:00 $\mathrm{h}(\mathrm{p}<0.05)$. However, there was no significant difference in reaction time between MEL and PLA at 12:00 $\mathrm{h}$ and 16:00 $\mathrm{h}(\mathrm{p}>0.05)$.

Table I. Mental performances (mean $\pm \mathrm{SE}$ ) measured at 08:00 h, 12:00 h, and 16:00 h after either MEL or PLA ingestion

\begin{tabular}{|l|c|c|c|c|c|c|}
\hline & \multicolumn{3}{|c|}{ PLA } & \multicolumn{3}{c|}{ MEL } \\
\hline Time of day & $8 \mathrm{~h}$ & $12 \mathrm{~h}$ & $16 \mathrm{~h}$ & $8 \mathrm{~h}$ & $12 \mathrm{~h}$ & $16 \mathrm{~h}$ \\
\hline Reaction time (s) & $0.49 \pm 0.06$ & $0.47 \pm 0.06^{*}$ & $0.45 \pm 0.05^{*}$ & $0.53 \pm 0.02^{\$}$ & $0.48 \pm 0.01^{*}$ & $0.46 \pm 0.02^{*}$ \\
\hline Vigilance test (UA) & $50.83 \pm 8.18$ & $56.25 \pm 6.05$ & $63.58 \pm 9.85^{*}$ & $38.5 \pm 6.44^{\$}$ & $50,14 \pm 6.89 *$ & $61.75 \pm 10.65^{*}$ \\
\hline
\end{tabular}

*: Significant difference at 12:00 $\mathrm{h}$ and 16:00 $\mathrm{h}$ in comparison with 08:00 $\mathrm{h}$ during the PLA and MEL session $(\mathrm{p}<0.05)$. \$: Significant difference between MEL and PLA at 08:00 h daytime $(\mathrm{p}<0.05)$. 
Concerning the vigilance test, there was a significant time-of-day $(\mathrm{F}=39.01 ; \mathrm{p}<0.05)$, treatment $(\mathrm{F}=7.54 ; \mathrm{p}<0.05)$ and interaction time-of-day $\times$ treatment effects. The post-hoc revealed that vigilance was higher at 16:00 h than 08:00 h during the PLA session $(\mathrm{p}<0.05)$. Moreover, measurements were significantly higher at 16:00 h than 12:00 h and 08:00 $\mathrm{h}$ with MEL. Furthermore, vigilance was significantly lower with MEL in comparison to the PLA at 08:00 $\mathrm{h}(\mathrm{p}<0.05)$. However, there was no significant difference between MEL and PLA session at 12:00 $\mathrm{h}$ and $16: 00 \mathrm{~h}$.

\section{Medicine ball throws (MBT)}

Main effects (time-of-day and treatment) were not significant for the MBT, interaction (treatment $\times$ time-of-day) effect being significant $(\mathrm{F}=6.22 ; \mathrm{p}<0.05)$. There were significantly lower MBT performances at 08:00 h, with MEL in comparison with PLA $(\mathrm{p}<0.05)$. There was no significant difference between MEL and PLA session at 12:00 $\mathrm{h}$ and 16:00 h (Fig. 2).

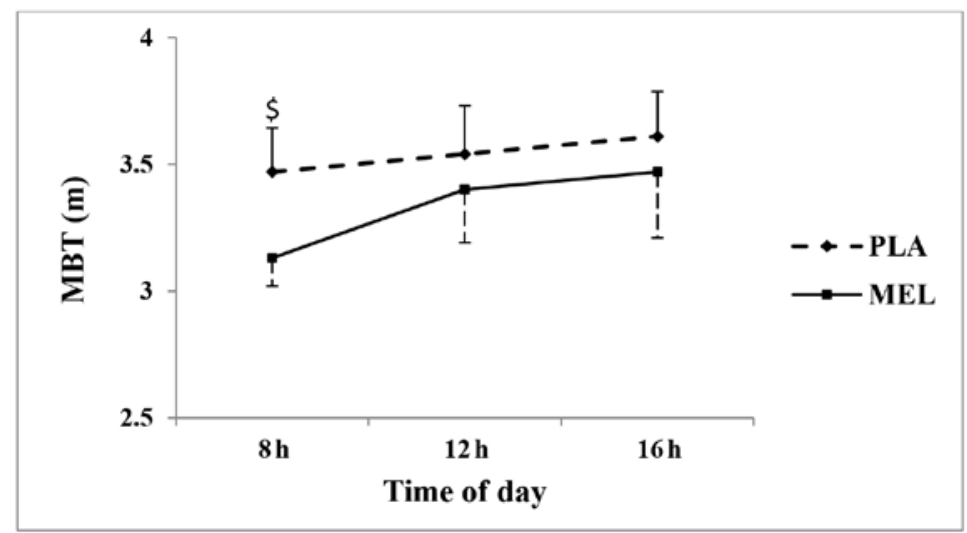

Fig. 2. Different performances of MBT (mean \pm SE) achieved at 08:00 h, 12:00 h and 16:00 h after either MEL or PLA ingestion in the morning. \$: Significant difference between MEL and PLA at 08:00 h daytime $(\mathrm{p}<0.05)$

\section{Five-jump test (5JT)}

Statistical analysis showed a significant daytime effect $(F=12.10 ; p<0.05)$ but the effect of treatment or interaction (treatment $\times$ time-of-day) was not significant. Performances in the 5JT were significantly higher at 16:00 h than 08:00 h $(\mathrm{p}<0.05)$ during MEL condition. However, there was no significant difference between MEL and PLA for any daytime (Fig. 3).

\section{Hand grip $(H G)$}

Statistical analysis showed a significant effect of dose $(\mathrm{F}=12.98 ; \mathrm{p}<0.05)$ and daytime $(\mathrm{F}=$ 10.56; $\mathrm{p}<0.05$ ) for HG measures. However, interaction (treatment $\times$ time-of-day) was not significant. HG performances were significantly higher at 16:00 $\mathrm{h}$ than 12:00 $\mathrm{h}$ and 08:00 $\mathrm{h}$ during MEL condition $(\mathrm{p}<0.05)$. However, HG was higher at 16:00h in comparison with 08:00 h during PLA session $(\mathrm{p}<0.05)$. Moreover, morning HG performances were lower with MEL $(\mathrm{p}<0.05)$ (Fig. 4). 


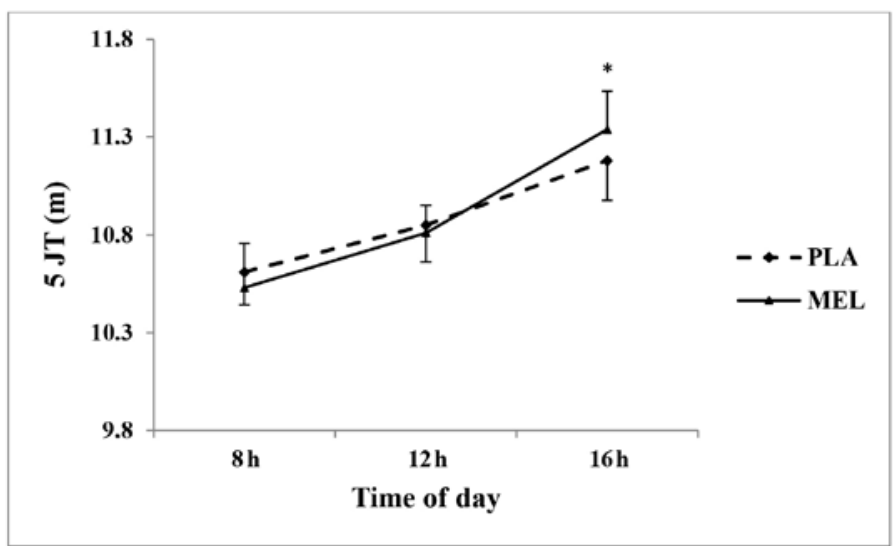

Fig. 3. Measures of $5 \mathrm{JT}$ (mean $\pm \mathrm{SE}$ ) achieved at 08:00 h, 12:00 $\mathrm{h}$ and 16:00 $\mathrm{h}$ after either MEL or PLA ingestion in the morning. *: Significant difference 16:00 $\mathrm{h}$ in comparison with 08:00 h during MEL session $(\mathrm{p}<0.05)$.

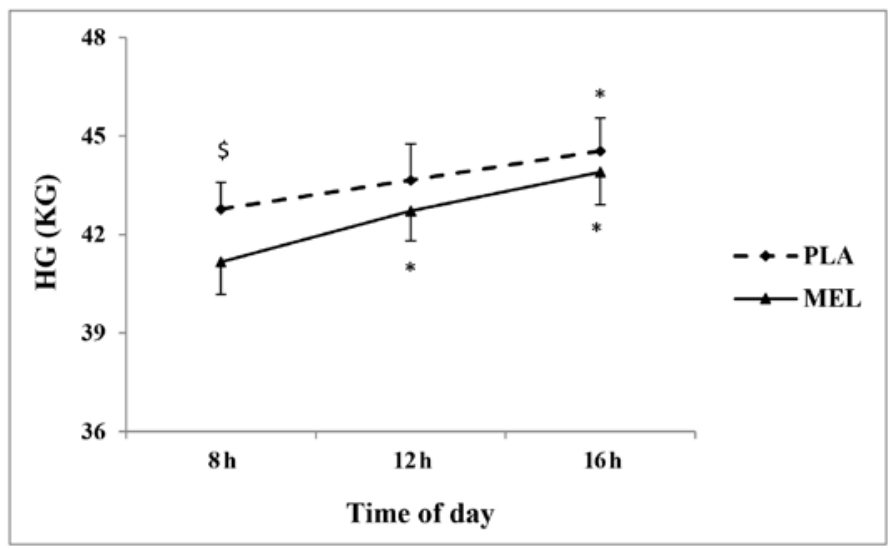

Fig. 4. Measures of HG (mean \pm SE) achieved at 08:00 h, 12:00 h and 16:00 h after either MEL or PLA ingestion in the morning. \$: Significant difference between PLA and MEL $(p<0.05)$ at 08:00 h. *: Significant difference between 16:00 $\mathrm{h}$ or 12:00 $\mathrm{h}$ and $08: 00 \mathrm{~h}(\mathrm{p}<0.05)$.

\section{Modified agility test (MAT)}

Statistical analysis showed a significant daytime effect $(F=4.8 ; \mathrm{p}<0.05)$, with no significant treatment and interaction (treatment $\times$ time-of-day) effects. There was a significant difference between 08:00 h, 12:00 $\mathrm{h}$ and 16:00 $\mathrm{h}$ with MEL, performances of agility being higher at 16:00 $\mathrm{h}$ than 12:00 $\mathrm{h}$ and 08:00 $\mathrm{h}(\mathrm{p}<0.05)$. However, there was no significant difference between MEL and PLA for any daytime (Fig. 5). 


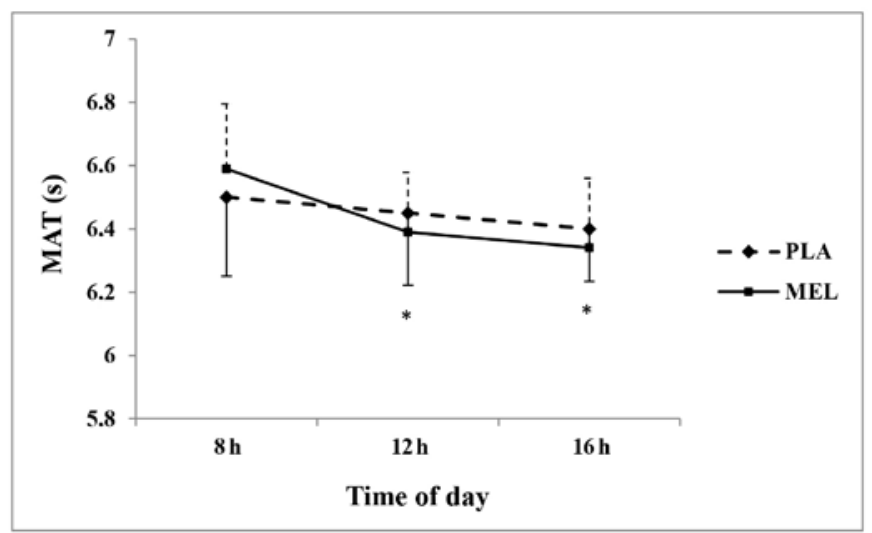

Fig. 5. Performances of modified agility test (mean \pm SE) achieved at 08:00 h, 12:00 h and 16:00 h after either MEL or PLA ingestion in the morning.

*: Significant difference between 16:00 $\mathrm{h}$ or 12:00 $\mathrm{h}$ and 08:00 $\mathrm{h}(\mathrm{p}<0.05)$

\section{Oral temperature:}

The GLM showed a significant effect of treatment and daytime $(\mathrm{F}=12.10 ; \mathrm{p}<0.05$ and $\mathrm{F}=$ $13.31 ; \mathrm{p}<0.05$, respectively). However, interaction (treatment $\times$ time-of-day) was not significant. Oral temperature was significantly higher at 16:00 h than 8:00 $\mathrm{h}(\mathrm{p}<0.05)$ during the two conditions. However, temperature were significantly higher with PLA in comparison with MEL at 08:00 h $(\mathrm{p}<0.05)$ (Fig. 6).

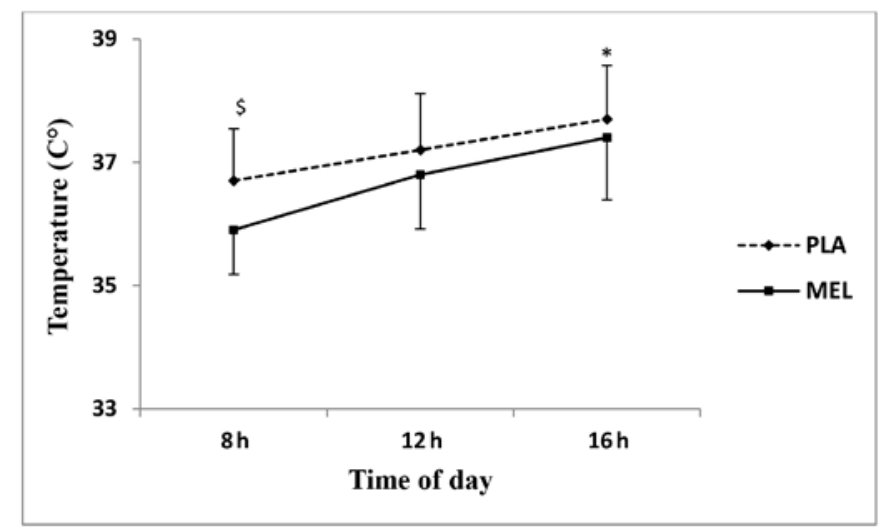

Fig. 6. Evolution of oral temperature (mean $\pm \mathrm{SE}$ ) measured at 08:00 h, 12:00 h and 16:00 h after either MEL or PLA ingestion in the morning. *: Significant difference between MEL and PLA at 08:00 $\mathrm{h}$ daytime $(\mathrm{p}<0.05)$. \$: Significant difference between 16:00 $\mathrm{h}$ and 08:00 h daytime $(\mathrm{p}<0.05)$.

\section{Discussion}

The aim of this study was to investigate the effect of morning MEL ingestion on the diurnal variation of short-term performances in teenager soccer players. The present findings showed that morning MEL ingestion affect morning performances (i.e., vigilance, reaction time, MBT, HG). However, performances measured in the afternoon remained unchanged. 
Concerning cognitive performances, the present study findings showed that morning measures of vigilance and simple reaction time were affected after morning MEL ingestion. Similarly, previous studies have shown that diurnal MEL ingestion affect essentially cognitive performances more than physical ones (6). For this reason, it should be noted that timing of MEL administration could influence specially the circadian rhythm of physiological parameters (17). In this way, morning MEL administration has been shown to phase delay circadian rhythms, while its administration in the late afternoon advanced it (19). Similarly, a dose-response relationship was established between MEL and decreased mental performances (8).

Moreover, the present findings indicate that reaction time and vigilance performances were significantly ameliorated at $16: 00 \mathrm{~h}$ in comparison with 08:00 $\mathrm{h}$ during the two conditions. However, there is no difference between the effect PLA and MEL on vigilance and reaction time at midday and in the afternoon. In this context, it is established that diurnal MEL ingestion may affect the ability of balance and coordination in young healthy subjects (9). Moreover, Jones (15) showed that MEL administration (5 mg) at 11:45 h affected choice reaction time, subjective alertness, short-term memory measured both at 13:00 h and 15:00 h. These results confirm the present study findings observed at 08:00 h. It seems that daytime MEL ingestion could affect cognitive performances and exert a hypnotic effect if it is very close to the evaluation. Furthermore, Atkinson et al. (5) showed that the diurnal ingestion of a lower dose of MEL (2.5 mg) at 11:45 h did not influence the subjective alertness and sleep before and after exercise done $75 \mathrm{~min}$ after MEL administration. Consequently, to assure that MEL does not affect the cognitive responses, it seems necessary to rise the duration between time of ingestion and the evaluation session.

Otherwise, the present study results indicated that all the selected performances were increased from the morning to the afternoon during the two conditions. It is well established that athletic performances display a diurnal pattern with higher values measured in the late afternoon and lower measures observed in the early morning (12). Likewise, it has been shown that soccer players perform at an optimum between 16:00 $\mathrm{h}$ and 20:00 $\mathrm{h}$ when soccerspecific skills and measures of physical performance are at their peak (18). In fact, increased body temperature through the day can be considered as a warm up; a decrease in muscle viscosity and increase in the velocity of nerve conduction and range of motion (6) and thus, an improvement in muscle performance accompany it. The enhancement of performances at midday can be explained with improved cognitive performance and core temperature at this time-point.

In addition, the present findings showed that morning MEL administration worsened short-term upper body performances in the morning but did not affect midday and afternoon measurements. Nevertheless, Atkinson et al. (3) concluded that daytime MEL ingestion did not affect $\mathrm{HG}$ and perceived exertion during a 4-km cycling time trial in physically active subjects. It should be declared that physical performances were measured at least 75 min after MEL ingestion in the above-mentioned study. Likewise, Antti et al. (1) have shown that MEL (6 mg) administration 1-h before performing a daytime resistance exercise session did not have any acute effects either on maximal jumping ability or muscle strength in spite of the well-known decrease in alertness. These previous studies confirm the present study findings when it comes to evaluate short-term lower performances (5JT and MAT). The deterioration of upper body performance with morning MEL ingestion can be explained also by the decrease of oral temperature observed in this condition. In the present study, MEL ingestion did not affect diurnal 5JT and MAT performances; measures being higher at 16:00 $\mathrm{h}$ 
in comparison with 12:00 h and at 12:00 h more than 08:00 $\mathrm{h}$. This improvement curve matched the increased temperature in the afternoon in comparison with the morning values during the two conditions. It should also be noted that there was a slight, but not significant, increase in 5JT performance at 16:00 h with MEL. This fact confirms previous findings that some effects of MEL werestill apparent 4-7 h after ingestion when its hypothermic effects had dissipated (3). Then, the present study findings are consistent with those of Atkinson et al (3) which concluded that the effects of diurnal MEL (5 mg) ingestion seem more pronounced for mental than for physical components of short-term athletic performance. Various factors may explain the discrepancy between the results of previous studies such as the time of ingestion, latency between ingestion and exercise, MEL dose, level of fitness, as well as the exercise intensity (4).

The discrepancy observed in the present study between cognitive and some physical performances after morning MEL ingestion could be attributed to the potential antioxidant effect of MEL. In fact, while daytime MEL ingestion has shown no beneficial effects on specific performance of soccer, recent studies have shown that diurnal MEL ingestion with a similar dose reduced oxidative stress, and improved immunological responses in soccer players (16). These findings could explain, at least in part, the present study results, about the slight but not significant positive effect of MEL upon 5JT and MAT performances. Moreover, from the results of the present study, we speculate that extending latency between MEL ingestion and physical exercise could attenuate the hypnotic effect associated to diurnal MEL ingestion.

A limitation of this study was that we did not measure the levels of MEL and oxidative stress biomarkers. Moreover, the sample size was small.

In conclusion, MEL ingested in the morning seems to affect morning cognitive performances and some short-term specific performances of soccer players and could ameliorate afternoon ones. MEL being a potent antioxidant may have therapeutic benefits in preventing exercise induced tissue damage and oxidative stress. In future studies, we will focus on the delayed effects of morning MEL ingestion upon redox status during evening exercise.

\section{REFERENCES}

1. Antti AM, Mika V, Juha JH, Petteri K, Atte VW: Effects of resistance exercise session after oral ingestion of melatonin on physiological and performance responses of adult men. Eur. J. App. Physiol. 96, 729-739 (2006)

2. Arendt J, Deacon S: Treatment of circadian rhythm disorders with melatonin. Chronobiol. Int. 14, 185-204 (1997)

3. Atkinson G, Jones H, Edwards BJ, Waterhouse JM: Effects of daytime ingestion of melatonin on short-term athletic performance. Ergonomics 48, 1512-1522 (2005)

4. Atkinson G, Buckley P, Edwards B, Reilly T, Waterhouse J: Are there hangover effects on physical performance when melatonin is ingested by athletes before nocturnal sleep? Int. J. Sports. Med. 22, 232-234 (2001)

5. Atkinson G, Holder A, Robertson C, Gant N, Drust B, Reilly T, Waterhouse J: Effects of melatonin on the thermoregulatory responses to intermittent exercise. J. Pineal Res. 39, 353-359 (2005)

6. Atkinson G, Drust B, Reilly T, Waterhouse J: Relevance of melatonin to sports medicine and science. Sports Med. 33, 809-831 (2003)

7. Bougard C, Bessot N, Moussay S, Sesboue B, Gauthier A: Effects of waking time and breakfast intake prior to evaluation of physical performance in the early morning. Chronobiol. Int. 26, 307-323 (2009)

8. Dollins AB, Lynch HJ, Wurtman RJ, Deng MH, Kischka KU, Gleason RE, Lieberman HR: Effect of pharmacological daytime doses of melatonin on human mood and performance. Psychopharmacology 11, 490496 (1993) 
9. Dollins AB, Zhdanova IV, Wurtman RJ: Effect of inducing nocturnal serum melatonin concentrations in daytime on sleep, mood, body temperature, and performance. Proc. Natl Acad. Sci. USA 91, 1824-1828 (1994)

10. Escames G, Ozturk G, Baño-Otálora B, Pozo MJ, Madrid JA, Reiter RJ: Exercise and melatonin in humans: reciprocal benefits. J. Pineal Res. 52, 1-11 (2012)

11. Ghattassi K, Graja A, Hammouda O, Chtourou H, Boudhina N, Chaouachi A, Souissi N: Effect of nocturnal melatonin ingestion on short-term anaerobic performance in soccer players. Biol. Rhythms Res. 45, 885-893 (2014)

12. Hammouda O, Chahed H, Chtourou H, Ferchichi S, Miled A, Souissi N: Morning to evening difference of biomarkers of muscle injury and antioxidant status in young trained soccer players. Bio. Rhythm 43, 431-438 (2012)

13. Horne JA, Ostberg O: A self-assessment questionnaire to determine morningness-eveningness in human circadian rhythms. Int. J. Chronobiol. 4, 97-110 (1976)

14. Hughes RJ, Badia P: Sleep-promoting and hypothermic effects of daytime melatonin administration in humans. Sleep 20, 124-131 (1997)

15. Jones $\mathrm{H}$ : The effect of daytime ingestion of melatonin on human performance. PhD Dissertation, Liverpool John Moores University, Liverpool. (2001)

16. Maldonado MD, Manfredi MJ, Ribas-Serna J, Garcia-Moreno H, Calvo JR: Melatonin administered immediately before an intense exercise reverses oxidative stress, improves immunological defenses and lipid metabolism in soccer players. Physiol. Behav. 105, 1099-1103 (2012)

17. Pitrosky B, Kirsch R, Malan A, Mocaer E, Pevet P: Organization of rat circadian rhythms during daily infusion of melatonin or S20098, a melatonin agonist. Am. J. Physiol. Regul. Integr. Comp. Physiol. 277, R812-R828 (1999)

18. Reilly T, Atkinson G, Edwards B, Waterhouse J, Farrelly K, Fairhurst E: Diurnal variation in temperature, mental and physical performance, and tasks specifically related to football (soccer). Chronobiol. Int. 24, 507519 (2007)

19. Rodriguez C, Mayo JC, Sainz RM, Antolin I, Herrera F, Martin V, Reiter RJ: Regulation of antioxidant enzymes: a significant role for melatonin. J. Pineal. Res. 36, 1-9 (2004)

20. Sassi RH, Dardouri W, Yahmed MH, Gmada N, Mahfoudhi ME, Gharbi Z: Relative and absolute reliability of a modified agility T-test and its relationship with vertical jump and straight sprint. J. Str. Cond. Res. 23, 16441651 (2009)

21. Tzischinski O, Lavie P: Melatonin possesses time-dependent hypnotic effects. Sleep 17, 638-645 (1994)

22. Vanecek J: Cellular mechanisms of melatonin action. Physiol. Rev. 78, 687-721 (1998)

23. Viola AU, Chellappa SL, Archer SN, Pugin F, Götz T, Dijk DJ, Cajochen C: Interindividual differences in circadian rhythmicity and sleep homeostasis in older people: effect of a PER3 polymorphism. Neurobiol. Aging 33, 17-27 (2012) 